\title{
Klinische Umsetzung der Leitlinien in Brustzentren
}

\author{
Gesprächsleitung: Matthias W. Beckmann, Erlangen \\ Teilnehmer: \\ Thomas Beck, Rosenheim
Christian Menzel, Salzburg \\ Daniel Fink, Zürich Rolf Kreienberg, Ulm
}

Frage 1: Die Behandlung von Frauen mit Mammakarzinom sollte in zertifizierten Brustzentren erfolgen.

Worin sehen Sie die Vorteile einer Versorgung in einem zertifizierten Brustzentrum?

Beck: Zertifizierte Brustzentren bieten in der Diagnostik und Therapie des Mammakarzinoms eine vergleichbare, garantierte Strukturqualität der interdisziplinären Versorgung, die durch die jährlichen Audits objektiv überprüfbar ist. Die Zentrumsbildung bewirkt eine Fallzahlsteigerung, die eine stetig wachsende Expertise aller Kooperationspartner zur Folge hat. Diese stetig wachsende Kompetenz in der Leistungserbringung kann auch unter ökonomischen Gesichtspunkten optimiert werden. Damit erhält die Mammakarzinompatientin im zertifizierten Brustzentrum maximale Behandlungsqualität zu möglichst günstigen Kosten im Hinblick auf die Leistungserbringung. Die Zertifizierung von Brustzentren hat nachhaltig zu einer flächendeckenden Qualitätsverbesserung der Mammakarzinombehandlung in Deutschland geführt.

Fink: Durch definierte Prozesse sowie kontinuierliche Messung der Qualitätsparameter wird die Behandlungsqualität verbessert. Zudem ermöglichen große Fallzahlen es dem ganzen Team des Brustzentrums, umfangreiche klinische Erfahrung zu sammeln.

Kreienberg: Die Vorteile für die Patientinnen bestehen darin, dass sie interdisziplinär von den verschiedensten Spezialisten auf dem höchsten Stand der Technik und auch des medizinischen Wissens behandelt werden. Zertifizierte Brustzentren verfügen über ein Qualitätsmanagementsystem, das sowohl die Strukturqualität als auch die Prozessqualität jährlich, die Ergebnisqualität in dreijährigen Abständen überprüft. Bisher ist es in der Medizin einmalig, dass Fachgesellschaften wie die Deutsche Krebsgesellschaft und die Deutsche Gesellschaft für
Senologie ein Zertifizierungssystem ins Leben gerufen haben, das Patientinnen die Transparenz ermöglicht, diese Ergebnisse zu erkennen und damit auf höchstem Niveau behandelt und betreut zu werden.

Menzel: Der Vorteil einer Versorgung von Frauen in einem zertifizierten Brustzentrum liegt vor allem darin, dass durch die Vorgabe und die jährliche Evaluierung der Leitlinien von Diagnostik und Therapie des Mammakarzinoms im Rahmen des Zertifizierungsverfahrens eine größtmögliche Garantie für ein optimales Qualitätsmanagement der Versorgung von an einem Mammakarzinom erkrankten Frauen bietet.

\section{Frage 2: Die Behandlung in einem zertifizierten Brustzentrum basiert auf Interdisziplinarität und festgeschriebenen Kooperationen. Sollte die Behandlung nach eigenen Leitlinien oder nach nationalen und internationalen Leitlinien erfolgen?}

Beck: Die nationalen und internationalen Leitlinien sind Grundlagen der Diagnostik und Therapie in zertifizierten Brustzentren. Sie sind wichtige «Spielregeln» der interdisziplinären Zusammenarbeit und müssen jeweils aktualisiert sein. Die Leitlinien dürfen aber nicht der weiteren Entwicklung der persönlichen Expertise in Teilbereichen hinderlich sein. Persönliche Erfahrungen mit speziellen Techniken oder Therapieerfahrungen unter kontrollierten Bedingungen müssen auch im zertifizierten Brustzentrum möglich bleiben.

Fink: Die Behandlung sollte nach nationalen / internationalen Leitlinien erfolgen, wobei man sich dabrüber im Klaren sein muss, dass diese Leitlinien immer einen Konsens darstellen und dabei breit gefasst sind. Daher sind möglichst detail-

\begin{tabular}{ll}
\hline KARGER & @ 2007 S. Karger GmbH, Freiburg \\
Fax +497614520714 & Accessible online at: \\
$\begin{array}{l}\text { E-mail Information@Karger.de } \\
\text { www.karger.com }\end{array}$ & www.karger.com/brc
\end{tabular}

Prof. Dr. med. Matthias W. Beckmann 
lierte eigene Richtlinien im Sinne eines «working protocol» für die täglichen Entscheidungen hilfreich.

Kreienberg: Wichtig ist, dass die Behandlung überhaupt nach Leitlinien erfolgt. In Deutschland verfügen wir über eine hochkarätige S 3-Leitlinie zum Thema «Mammakarzinom», nach der sich alle Brustzentren richten müssen. Daneben sind die aktuellen Behandlungsempfehlungen der Arbeitsgemeinschaft Gynäkologische Onkologie, die in jährlichen Abständen aktualisiert werden, zusätzlich hilfreich für die Behandlung der Patientinnen. Andere nationale und internationale Leitlinien werden nicht benötigt, da die Daten aus diesen Leitlinien sowohl in den aktuellen Behandlungsempfehlungen der AGO als auch in der S 3-Leitlinie Mammakarzinom mit berücksichtigt sind.

Menzel: Die Behandlung sollte nach nationalen, gegebenenfalls auch internationalen (EUSOMA-) Leitlinien erfolgen.

Frage 3: Im Rahmen der interdisziplinären Tumorkonferenz wird eine Leitlinien-orientiere Therapieempfehlung formuliert. Bei wie viel Prozent der Patientinnen in einem zertifizierten Brustzentrum erfolgt eine Leitlinien-konforme Behandlung? Was sind die Hauptgründe für eine nicht Leitlinien-konforme Behandlung?

Beck: Die Tumorkonferenz formuliert eine leitlinienkonforme Therapieempfehlung für den individuellen Fall. Abweichungen von der Leitlinie ergeben sich durch Grenzindikationen oder Kontraindikationen. Hauptproblem sind die Gültigkeit der Leitlinien für sehr alte Patientinnen (>80 Jahre) und Patientinnen mit reduziertem Allgemeinzustand, Multimorbidität usw. Insoweit ist die Abweichung von der Leitlinie ärztlich begründbar und nachvollziehbar. Dies betrifft zirka 10\% unseres Patientenkollektives. Darüber hinaus führt die fehlende Zustimmung der Patientin zur vorgeschlagenen Therapie $\mathrm{zu}$ weiteren 5\% Abweichungen von der leitlinienkonformen Therapieempfehlung. Die Abweichungen von der Leitlinie sind zu dokumentieren.

Fink: Der Prozentsatz an Leitlinien-konformen Behandlungen sollte mehr als $85 \%$ betragen. Wichtigste Gründe für nicht Leitlinien-konforme Behandlungen sind Ablehnung durch die Patientin bzw. schwerwiegende Nebendiagnosen.

Kreienberg: Zirka 70-80\% der Patientinnen werden in allen Behandlungsschritten leitliniengerecht behandelt. Angestrebt werden immer $100 \%$. Gründe für eine nicht leitlinienkonforme Behandlung können fortgeschrittenes Alter der Patientin, Komorbidität, Unverträglichkeiten verschiedenster Therapieformen und nicht zuletzt der Wunsch der Patientin, nicht die vorgeschlagene Therapie durchzuführen, sein.
Menzel: Es sollte bei mindestens $90 \%$ der Patientinnen eine Leitlinien-konforme Behandlung durchgeführt werden. Die Hauptgründe für die nicht Leitlinien-konforme Behandlung bestehen darin, dass die Therapie durch das Vorliegen spezieller Umstände wie z.B. Polymorbidität, Alter oder Patientenwunsch individualisiert und somit nicht Leitlinien-konform durchgeführt werden muss. An unserem Brustzentrum werden derzeit jährlich knapp 400 Mammakarzinom-Patientinnen (Neuerkrankungen) betreut, somit wird in zirka 20 Fällen bei der Behandlung nicht Leitlinien-konform vorgegangen.

\section{Frage 4: Die derzeit vorhandenen S 3-Leitlinien zur Früherkennung, Diagnostik und Therapie des Mamma- karzinoms werden alle 3 Jahre überarbeitet. Wie werden neue Erkenntnisse in Ihrem Brustzentrum implementiert, die sich in der Zwischenzeit, also während der Überarbeitung, ergeben?}

Beck: Die S 3-Leitlinie ist in der Zwischenzeit in mehreren Positionen nicht mehr aktuell (Version 2004), so dass sie aufgrund von Fortschritten in Diagnostik und Therapie überarbeitet werden muss. Die Therapieentscheidungen im individuellen Fall müssen in der Zwischenzeit an die Ergebnisse neuerer Empfehlungen adaptiert werden. Ferner sollten die Patienten in randomisierte Studien eingebracht werden, um die Generierung neuer Erkenntnisse weiter voranzutreiben.

Fink: Unser «working protocol» wird jeweils bei neuen Erkenntnissen angepasst. Dies kann aufgrund des ASCO-Meetings, der San Antonio Breast Cancer Conference und/oder des St. Gallen-Meetings erfolgen.

Kreienberg: Wie schon ausgeführt, werden S 3-Leitlinien in dreijährigen Abständen, die aktuellen Behandlungsempfehlungen der Arbeitsgemeinschaft Gynäkologische Onkologie in jährlichen Abständen überarbeitet. Hinzu kommen die Erkenntnisse aus den aktuellen Kongressen wie St. Gallen, San Antonio und dem ASCO, die in den Brustzentren üblicherweise sehr genau beobachtet und beachtet werden. Auf der Grundlage der neuesten Kongressinformationen und der nachfolgenden Expertenbeurteilungen sowie der jährlichen aktuellen Behandlungsempfehlungen der AGO können die Brustzentren auf dem neuesten Stand der Literatur therapieren. Die S 3-Leitlinie ist dann der größere Rahmen, der in dreijährigen Abständen überarbeitet wird. Insgesamt ist festzustellen, dass - wenn alle zertifizierten Brustzentren in allen Fällen sich ausschließlich an die S 3-Leitlinie halten würden der Verlust durch die nur dreijährige Überarbeitung außerordentlich gering wäre. Darüber hinaus ist geplant, die S 3-Leitlinie nicht mehr in dreijährigen Abständen zu aktualisieren, sondern - zumindest in der Internet-Version - kontinuierlich an die neuesten Ergebnisse von Metaanalysen und publizierte Studien anzupassen. 
Menzel: Während der Zeit der Überarbeitung der S 3-Leitlinien zur Früherkennung und Diagnostik sowie Therapie des Mammakarzinoms werden Neuerkenntnisse nur dann implementiert, wenn neue Studien für Neubehandlungsrichtlinien signifikante Ergebnisse aufzeigen und die Beibehaltung eines «alten» Behandlungsschemas zum Nachteil der Patientin wäre.

\section{Frage 5: Leitlinien sind Handlungsempfehlungen in speziellen Situationen der Diagnostik und Therapie. Glauben Sie, dass die Implementierung der Leitlinien 2002 zu einer Veränderung der Versorgung der Patientinnen geführt hat?}

Beck: Die S3-Leitlinie von 2004 sind derzeit die wichtigsten Handlungsempfehlungen für zertifizierte Brustzentren. Sie haben zu einer Vereinheitlichung der Standards in Diagnostik und Therapie geführt, so dass Qualitätsunterschiede auf hohem Niveau egalisiert wurden. Wünschenswert wären aus meiner Sicht Konkretisierungen in Teilbereichen wie z.B. die kritische Indikationsstellung plastisch/chirurgischer Sofortrekonstruktionen.

Fink: Die Implementierung der Leitlinien 2002 hat in unserem Brustzentrum nicht zu einer großen Veränderung der Versorgung unserer Patientinnen geführt, da unser «working protocol» aufgrund der Anpassung bei neuen Erkenntnissen etwa auf diesem Level war. Die Leitlinien 2002 haben uns gezeigt, dass unser EUSOMA-zertifiziertes Brustzentrum den deutschen Richtlinien durchaus genügt.

Kreienberg: Ich glaube, dass die Implementierung der Leitlinie und der dort formulierten klaren Handlungsempfehlungen zu einer deutlichen, flächendeckenden Verbesserung in der Diagnostik und Therapie unserer Patientinnen geführt hat. Flankiert wurde diese Maßnahme sicher durch die Ein- führung der zertifizierten Brustzentren, mit der Einführung des Qualitätsmanagement-Systems und der jährlichen Kontrolle der Struktur-, Prozess- und jetzt auch der Ergebnisqualität. Beides zusammen hat sicher zu erheblichen Verbesserungen geführt und geholfen, Unter- und Übertherapien $\mathrm{zu}$ vermeiden.

Menzel: Die Implementierung der Leitlinien 2002 hat in unserem Brustzentrum ohne Zweifel zu einer Veränderung der Versorgung der Patientinnen geführt.

\section{Teilnehmer}

Prof. Dr. Thomas Beck

Klinik für Gynäkologie und Geburtshilfe

Klinikum Rosenheim

Pettenkoferstr. 10

83022 Rosenheim, Deutschland

Tel. +49 8031-363252, Fax -364932

E-Mail gynaekologie@kliro.de

Prof. Dr. Rolf Kreienberg

Universitäts-Frauenklinik

Prittwitzstr. 43

89070 Ulm, Deutschland

Tel. +49 731 500-58501

E-Mail rolf.kreienberg@medizin.uni-ulm.de

Prof. Dr. Daniel Fink

UniversitätsSpital Zürich

Klinik für Gynäkologie

Frauenklinikstrasse 10

8091 Zürich, Schweiz

Tel. +41 44 255-5200, Fax -4433

E-Mail daniel.fink@usz.ch

Hofrat Prim. Prof. Dr. Christian Menzel

Universitätsklinik für Spezielle Gynäkologie

Müllner Hauptstraße 48

5020 Salzburg, Österreich

Tel. +43 662 4482-2571, Fax -2572

E-Mail b.funke@salk.at 